(C) 2003 American Chemical Society, Org. Lett., Frederick ol030061c Supporting Info Page 1

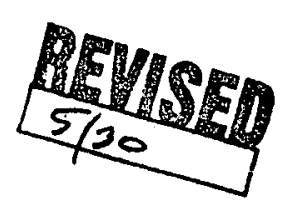

SUPPLEMENTARY MATERIALS

for

the communication

entitled

\title{
A Highly Stereoselective Saucy-Marbet Rearrangement Using Chiral Ynamides. Synthesis of Highly Substituted Chiral Homoallenyl Alcohols.
}

authored by

Michael O. Frederick, Richard P. Hsung*, Robert H. Lambeth, Jason A. Mulder, and Michael R. Tracey

Department of Chemistry, University of Minnesota, Minneapolis, MN 55455 
(C) 2003 American Chemical Society, Org. Lett., Frederick ol030061c Supporting Info Page 2

\section{General procedure for propargyl alcohol addition / Saucy-Marbet rearrangement:}

Ynamide $(0.2 \mathrm{mmol})$, anhy $p$-nitrobenzenesulfonic acid $(0.2$ equiv), propargyl alcohol (1-2 equiv), and anhydrous toluene $(4 \mathrm{~mL})$ were combined in a flame-dried $25 \mathrm{~mL}$ sealed tube under nitrogen atmosphere. The tube was sealed and the reaction mixture was heated at $100{ }^{\circ} \mathrm{C}$ for $24-48 \mathrm{~h}$. The reaction was followed with TLC and/or LCMS analysis. Once completed by TLC analysis, the reaction was cooled to room temperature, filtered through celite, and concentrated in vacuo. The residue was purified by flash silica gel column chromatography (gradient: $0 \%$ to $25 \%$ EtOAc in hexanes) to provide the rearranged products in yields indicated in the text.

\section{General procedure for reductive removal of chiral auxiliary}

Allene $(0.1 \mathrm{mmol}), \mathrm{LiBH}_{4}$ (3 equiv), and anhy THF $(10 \mathrm{~mL})$ were combined in a flame-dried RB-flask. The reaction mixture was allowed to stir overnight at $\mathrm{rt}$. The reaction was quenched with $\mathrm{H}_{2} \mathrm{O}(10 \mathrm{~mL})$ extracted with ether $(3 \times 5 \mathrm{~mL})$, dried over $\mathrm{Na}_{2} \mathrm{SO}_{4}$, and concentrated in vacuo. The residue was purified by flash silica gel column chromatography (gradient: $0 \%$ to $25 \%$ EtOAc in hexanes) to provide homo allenyl alcohols in yields as indicated in the text.

\section{Allene 9}

$\mathrm{R}_{\mathrm{r}}=0.38\left[25 \%\right.$ EtOAc in hexanes]; clear oil; $[\alpha]_{\mathrm{D}}{ }^{23}-68.2\left(\mathrm{c} 0.51, \mathrm{CH}_{2} \mathrm{Cl}_{2}\right) ;{ }^{1} \mathrm{H}$ NMR $\left(500 \mathrm{MHz}, \mathrm{CDCl}_{3}\right) \delta 1.62$ (dd, $3 \mathrm{H}, J=3.0,7.0 \mathrm{~Hz}), 3.66(\mathrm{ddq}, 2 \mathrm{H}, J=2.5,7.0,18.0 \mathrm{~Hz}), 4.31(\mathrm{dd}, 1 \mathrm{H}, J=3.5,9.0 \mathrm{~Hz}), 4.71(\mathrm{t}, 1 \mathrm{H}, J=$ $9.0 \mathrm{~Hz}$ ), 5.09-5.16 (m, $1 \mathrm{H}), 5.21(\mathrm{dddd}, 1 \mathrm{H}, J=2.5,3.0,7.0,7.0), 5.44(\mathrm{dd}, 1 \mathrm{H}, J=3.5,9.0 \mathrm{~Hz}), 7.27-7.47$ $(\mathrm{m}, 5 \mathrm{H}) ;{ }^{13} \mathrm{C}$ NMR $\left(75 \mathrm{MHz} \mathrm{CDCl}_{3}\right) \delta 13.9,36.1,57.5,70.0,82.5,86.7,125.9,128.6,129.0,138.7,153.2$, 170.4, 205.9; IR (thin film) $\mathrm{cm}^{-1} 2970(\mathrm{~m}), 2961(\mathrm{~m}), 2925(\mathrm{~m}), 2870(\mathrm{w}), 1776(\mathrm{~s}), 1705(\mathrm{~s})$; mass spectrum (APCI): $\mathrm{m} / \mathrm{e}$ (\% relative intensity) $258(23)(\mathrm{M}+\mathrm{H})^{+}, 164(28), 146(26), 120(100), 95(92), 87$ (21).

Allene 10

$\mathrm{R}_{\mathrm{f}}=0.38$ [25\% EtOAc in hexanes]; clear oil; $[\alpha]_{\mathrm{D}}{ }^{23}-51.6\left(\mathrm{c} 0.60, \mathrm{CH}_{2} \mathrm{Cl}_{2}\right) ;{ }^{1} \mathrm{H} \mathrm{NMR}\left(500 \mathrm{MHz}, \mathrm{CDCl}_{3}\right) \delta 1.62$ $(\mathrm{dd}, 3 \mathrm{H}, J=3.0,7.0 \mathrm{~Hz}), 3.66(\mathrm{ddq}, 2 \mathrm{H}, J=2.5,7.0,18.0 \mathrm{~Hz}), 4.31(\mathrm{dd}, 1 \mathrm{H}, J=3.5,9.0 \mathrm{~Hz}), 4.71(\mathrm{t}, 1 \mathrm{H}, J=$ $9.0 \mathrm{~Hz}$ ), 5.09-5.16 (m, $1 \mathrm{H}$ ), 5.21 (dddd, $1 \mathrm{H}, J=2.5,3.0,7.0,7.0$ ), 5.44 (dd, $1 \mathrm{H}, J=3.5,9.0 \mathrm{~Hz}$ ), 7.27-7.47 $(\mathrm{m}, 5 \mathrm{H}) ;{ }^{13} \mathrm{C}$ NMR $\left(75 \mathrm{MHz}, \mathrm{CDCl}_{3}\right) \delta 13.9,36.1,57.5,70.0,82.5,86.7,125.9,128.6,129.0,138.7,153.2$, 170.4, 205.9; IR (thin film) $\mathrm{cm}^{-1} 2970(\mathrm{~m}), 2961(\mathrm{~m}), 2925(\mathrm{~m}), 2870(\mathrm{w}), 1776(\mathrm{~s}), 1705$ (s); mass spectrum (APCI): m/e (\% relative intensity) $258(23)(\mathrm{M}+\mathrm{H})^{+}, 164(28), 146(26), 120(100), 95$ (92), 87 (21).

\section{Allene 12}

$\mathrm{R}_{\mathrm{f}}=0.42\left[25 \%\right.$ EtOAc in hexanes]; $\mathrm{mp}=92-94^{\circ} \mathrm{C} ;[\alpha]_{\mathrm{D}}{ }^{23}-122.0\left(\mathrm{c} 0.91, \mathrm{CH}_{2} \mathrm{Cl}_{2}\right) ;{ }^{1} \mathrm{H}$ NMR $\left(500 \mathrm{MHz}, \mathrm{CDCl}_{3}\right)$ $\delta$ 0.86-0.89 (m, $3 \mathrm{H}), 1.25-1.32(\mathrm{~m}, 4 \mathrm{H}), 1.49-1.56(\mathrm{~m}, 1 \mathrm{H}), 1.70(\mathrm{dd}, 3 \mathrm{H}, J=3.0,7.0 \mathrm{~Hz}), 1.71-1.77(\mathrm{~m}, 1$ $\mathrm{H}), 4.23(\mathrm{dq}, 1 \mathrm{H}, J=1.0,7.5 \mathrm{~Hz}), 4.41(\mathrm{dd}, 1 \mathrm{H}, J=3.0,9.0 \mathrm{~Hz}), 4.44(\mathrm{t}, 1 \mathrm{H}, J=9.0 \mathrm{~Hz}), 4.72(\mathrm{~d}, 1 \mathrm{H}, J=5.5$ $\mathrm{Hz}$ ), 5.05 (ddt, $1 \mathrm{H}, J=3.5,7.5,11.5 \mathrm{~Hz}$ ), 5.22 (ddq, $1 \mathrm{H}, J=2.0,7.5,7.5 \mathrm{~Hz}$ ), 5.34 (ddd, $1 \mathrm{H}, J=3.5,5.5,8.5$ $\mathrm{Hz})$, 7.10-7.15 (m, $4 \mathrm{H}), 7.25-7.33(\mathrm{~m}, 6 \mathrm{H}) ;{ }^{13} \mathrm{C} \mathrm{NMR}\left(75 \mathrm{MHz}, \mathrm{CDCl}_{3}\right) \delta 13.9,14.2,29.1,31.0,43.3,50.4$, $56.2,64.5,87.3,89.0,126.9,127.7,128.2,128.6,128.7,129.4,137.8,139.4,152.8,174.1,205.4$ (missing 1 signal due to overlap of the terminal allene carbons); IR (thin film) $\mathrm{cm}^{-1} 2956(\mathrm{~m}), 2922(\mathrm{~m}), 2854(\mathrm{w}), 1781$ (s), 1691 (s); mass spectrum (APCI): m/e (\% relative intensity) 404 (75) $(\mathrm{M}+\mathrm{H})^{+}, 378(21), 360$ (20), 254 (33), $193(18), 151(100)$.

\section{Allene 13}

$\mathrm{R}_{\mathrm{f}}=0.42$ [25\% EtOAc in hexanes]; clear oil; ' $\mathrm{H}$ NMR $\left(500 \mathrm{MHz}, \mathrm{CDCl}_{3}\right)$ Major isomer: $\delta 0.86-0.89(\mathrm{~m}, 3 \mathrm{H})$, $1.24-1.32(\mathrm{~m}, 4 \mathrm{H}), 1.51-1.59(\mathrm{~m}, 1 \mathrm{H}), 1.70(\mathrm{dd}, 3 \mathrm{H}, J=3.0,7.0 \mathrm{~Hz}), 1.71-1.78(\mathrm{~m}, 1 \mathrm{H}), 4.15(\mathrm{dq}, 1 \mathrm{H}, J=$ $1.0,7.5 \mathrm{~Hz}), 4.36-4.46(\mathrm{~m}, 2 \mathrm{H}), 4.74(\mathrm{~d}, 1 \mathrm{H}, J=5.0 \mathrm{~Hz}), 5.08-5.14(\mathrm{~m}, 1 \mathrm{H}), 5.20(\mathrm{ddq}, 1 \mathrm{H}, J=2.0,7.5,7.5$ $\mathrm{Hz}), 5.30-5.34(\mathrm{~m}, 1 \mathrm{H}), 7.10-7.15(\mathrm{~m}, 4 \mathrm{H}), 7.25-7.33(\mathrm{~m}, 6 \mathrm{H})$; Minor isomer: $\delta 0.86-0.89(\mathrm{~m}, 3 \mathrm{H}), 1.24-1.32$ $(\mathrm{m}, 4 \mathrm{H}), 1.51-1.59(\mathrm{~m}, 1 \mathrm{H}), 1.63(\mathrm{dd}, 3 \mathrm{H}, J=3.0,7.0 \mathrm{~Hz}), 1.71-1.78(\mathrm{~m}, 1 \mathrm{H}), 4.10(\mathrm{dq}, 1 \mathrm{H}, J=1.0,7.5 \mathrm{~Hz})$, 
(C) 2003 American Chemical Society, Org. Lett., Frederick ol030061c Supporting Info Page 3

$4.36-4.46(\mathrm{~m}, 2 \mathrm{H}), 4.69(\mathrm{~d}, 1 \mathrm{H}, J=5.0 \mathrm{~Hz}), 5.08-5.14(\mathrm{~m}, 1 \mathrm{H}), 5.20(\mathrm{ddq}, 1 \mathrm{H}, J=2.0,7.5,7.5 \mathrm{~Hz}), 5.30-5.34$ $(\mathrm{m}, 1 \mathrm{H}), 7.10-7.15(\mathrm{~m}, 4 \mathrm{H}), 7.25-7.33(\mathrm{~m}, 6 \mathrm{H})$; IR (thin film) $\mathrm{cm}^{-1} 2956(\mathrm{~m}), 2922(\mathrm{~m}), 2854(\mathrm{w}), 1781(\mathrm{~s})$, 1691 (s); mass spectrum (APCI): m/e (\% relative intensity) $404(75)(\mathrm{M}+\mathrm{H})^{+}, 378(21), 360(20), 254(33), 193$ (18), $151(100)$.

\section{Allene 21}

$\mathrm{R}_{\mathrm{f}}=0.41\left[25 \%\right.$ EtOAc in hexanes]; $\mathrm{mp}=74-75^{\circ} \mathrm{C} ;[\alpha]_{\mathrm{D}}{ }^{23}-16.7\left(\mathrm{c} 1.26, \mathrm{CH}_{2} \mathrm{Cl}_{2}\right) ;{ }^{\prime} \mathrm{H} \mathrm{NMR}\left(500 \mathrm{MHz}, \mathrm{CDCl}_{3}\right)$ $\delta 0.89(\mathrm{~m}, 3 \mathrm{H}), 1.25-1.27(\mathrm{~m}, 4 \mathrm{H}), 1.51(\mathrm{dd}, 3 \mathrm{H}, J=3.5,7.0 \mathrm{~Hz}), 1.53-1.57(\mathrm{~m}, 1 \mathrm{H}), 1.73-1.80(\mathrm{~m}, 1 \mathrm{H}), 4.26$ $(\mathrm{dd}, 1 \mathrm{H}, J=4.5,9.0 \mathrm{~Hz}), 4.26-4.30(\mathrm{~m}, 1 \mathrm{H}), 4.69(\mathrm{t}, 1 \mathrm{H}, J=9.0 \mathrm{~Hz}), 5.11-5.17(\mathrm{~m}, 2 \mathrm{H}), 5.45(\mathrm{dd}, 1 \mathrm{H}, J=$ 4.5, $9.0 \mathrm{~Hz}$ ), 7.29-7.39 (m, $5 \mathrm{H}) ;{ }^{13} \mathrm{C}$ NMR $\left(75 \mathrm{MHz} \mathrm{CDCl}_{3}\right) \delta 13.9,22.4,29.2,30.9,43.2,57.7,69.7,87.7$, $88.6,125.8,128.5,129.0,138.8,153.1,173.4,205.3$ (missing 1 signal due to overlap of the terminal allene carbons); IR (thin film) $\mathrm{cm}^{-1} 2960(\mathrm{~m}), 2927(\mathrm{~m}), 2859(\mathrm{w}), 1780(\mathrm{~s}), 1704(\mathrm{~s})$; mass spectrum (APCI): m/e (\% relative intensity) $314(33)(\mathrm{M}+\mathrm{H})^{+}, 270(15), 164(20), 151(100), 123(36), 120$ (46).

\section{Allene ent-21}

$\mathrm{R}_{\mathrm{f}}=0.41\left[25 \%\right.$ EtOAc in hexanes]; $\mathrm{mp}=74-75^{\circ} \mathrm{C} ;[\alpha]_{\mathrm{D}}{ }^{23}+16.7\left(\mathrm{c} 0.24, \mathrm{CH}_{2} \mathrm{Cl}_{2}\right) ;{ }^{\prime} \mathrm{H} \mathrm{NMR}\left(500 \mathrm{MHz}, \mathrm{CDCl}_{3}\right)$ $\delta 0.89(\mathrm{~m}, 3 \mathrm{H}), 1.25-1.27(\mathrm{~m}, 4 \mathrm{H}), 1.51(\mathrm{dd}, 3 \mathrm{H}, J=3.5,7.0 \mathrm{~Hz}), 1.53-1.57(\mathrm{~m}, 1 \mathrm{H}), 1.73-1.80(\mathrm{~m}, 1 \mathrm{H}), 4.26$ $(\mathrm{dd}, 1 \mathrm{H}, J=4.5,9.0 \mathrm{~Hz}), 4.26-4.30(\mathrm{~m}, 1 \mathrm{H}), 4.69(\mathrm{t}, 1 \mathrm{H}, J=9.0 \mathrm{~Hz}), 5.11-5.17(\mathrm{~m}, 2 \mathrm{H}), 5.45(\mathrm{dd}, 1 \mathrm{H}, J=$ 4.5, $9.0 \mathrm{~Hz}), 7.29-7.39(\mathrm{~m}, 5 \mathrm{H}) ;{ }^{13} \mathrm{C}$ NMR $\left(75 \mathrm{MHz}, \mathrm{CDCl}_{3}\right) \delta$ 13.9, 22.4, 29.2, 30.9, 43.2, 57.7, 69.7, 87.7, $88.6,125.8,128.5,129.0,138.8,153.1,173.4,205.3$ (missing 1 signal due to overlap of the terminal allene carbons); IR (thin film) $\mathrm{cm}^{-1} 2960(\mathrm{~m}), 2927$ (m), 2859 (w), 1780 (s), 1704 (s); mass spectrum (APCI): m/e (\% relative intensity) $314(33)(\mathrm{M}+\mathrm{H})^{+}, 270$ (15), $164(20), 151(100), 123(36), 120$ (46).

\section{Allene 22}

$\mathrm{R}_{\mathrm{f}}=0.39\left[25 \%\right.$ EtOAc in hexanes]; $\mathrm{mp}=50-51^{\circ} \mathrm{C} ;[\alpha]_{\mathrm{D}}{ }^{23}-18.4\left(\mathrm{c} 0.38, \mathrm{CH}_{2} \mathrm{Cl}_{2}\right) ;{ }^{1} \mathrm{H} \mathrm{NMR}\left(500 \mathrm{MHz}, \mathrm{CDCl}_{3}\right)$ $\delta 0.87(\mathrm{t}, 3 \mathrm{H}, J=7.0 \mathrm{~Hz}), 0.90(\mathrm{t}, 3 \mathrm{H}, J=7.0 \mathrm{~Hz}), 1.22-1.25(\mathrm{~m}, 4 \mathrm{H}), 1.26-1.36(\mathrm{~m}, 6 \mathrm{H}), 1.52-1.56(\mathrm{~m}, 1 \mathrm{H})$, $1.74-1.79(\mathrm{~m}, 1 \mathrm{H}), 1.84-1.89(\mathrm{~m}, 2 \mathrm{H}), 4.25(\mathrm{dd}, 1 \mathrm{H}, J=4.0,9.0 \mathrm{~Hz}), 4.29(\mathrm{dq}, 1 \mathrm{H}, J=2.5,8.0 \mathrm{~Hz}), 4.69(\mathrm{t}, 1$ $\mathrm{H}, J=9.0 \mathrm{~Hz}), 5.14-5.19(\mathrm{~m}, 2 \mathrm{H}), 5.45(\mathrm{dd}, 1 \mathrm{H}, J=4.0,9.0 \mathrm{~Hz}), 7.14-7.39(\mathrm{~m}, 5 \mathrm{H}) ;{ }^{13} \mathrm{C} \mathrm{NMR}(75 \mathrm{MHz}$, $\left.\mathrm{CDCl}_{3}\right) \delta 13.8,13.9,22.3,22.4,28.4,28.6,29.2,30.8,31.1,43.3,57.6,69.6,89.1,93.1,125.7,128.4,128.9$, 138.7, 153.1, 173.4, 204.5; IR (thin film) $\mathrm{cm}^{-1} 2965(\mathrm{~m}), 2930(\mathrm{~m}), 2925(\mathrm{~m}), 2860(\mathrm{w}), 1777(\mathrm{~s}), 1702(\mathrm{~s})$; mass spectrum (APCI): m/e (\% relative intensity) $370(40)(M+H)^{+}, 326(7), 207(100), 164(6), 120$ (12).

\section{Allene ent-22}

$\mathrm{R}_{\mathrm{f}}=0.39\left[25 \%\right.$ EtOAc in hexanes]; $\mathrm{mp}=52-53^{\circ} \mathrm{C} ;[\alpha]_{\mathrm{D}}^{23}+13.0\left(\mathrm{c} 0.40, \mathrm{CH}_{2} \mathrm{Cl}_{2}\right) ;{ }^{\prime} \mathrm{H} \mathrm{NMR}\left(500 \mathrm{MHz}, \mathrm{CDCl}_{3}\right)$ $\delta 0.87(\mathrm{t}, 3 \mathrm{H}, J=7.0 \mathrm{~Hz}), 0.90(\mathrm{t}, 3 \mathrm{H}, J=7.0 \mathrm{~Hz}), 1.22-1.25(\mathrm{~m}, 4 \mathrm{H}), 1.26-1.36(\mathrm{~m}, 6 \mathrm{H}), 1.52-1.56(\mathrm{~m}, 1 \mathrm{H})$, $1.74-1.79(\mathrm{~m}, 1 \mathrm{H}), 1.84-1.89(\mathrm{~m}, 2 \mathrm{H}), 4.25(\mathrm{dd}, 1 \mathrm{H}, J=4.0,9.0 \mathrm{~Hz}), 4.29(\mathrm{dq}, 1 \mathrm{H}, J=2.5,8.0 \mathrm{~Hz}), 4.69(\mathrm{t}, 1$ $\mathrm{H}, J=9.0 \mathrm{~Hz}), 5.14-5.19(\mathrm{~m}, 2 \mathrm{H}), 5.45(\mathrm{dd}, 1 \mathrm{H}, J=4.0,9.0 \mathrm{~Hz}), 7.14-7.39(\mathrm{~m}, 5 \mathrm{H}) ;{ }^{13} \mathrm{C} \mathrm{NMR}(75 \mathrm{MHz}$, $\left.\mathrm{CDCl}_{3}\right) \delta 13.8,13.9,22.3,22.4,28.4,28.6,29.2,30.8,31.1,43.3,57.6,69.6,89.1,93.1,125.7,128.4,128.9$, 138.7, 153.1, 173.4, 204.5 ; IR (thin film) $\mathrm{cm}^{-1} 2965$ (m), 2930 (m), 2925 (m), 2860 (w), 1777 (s), 1702 (s); mass spectrum (APCI): $\mathrm{m} / \mathrm{e}$ (\% relative intensity) $370(40)(\mathrm{M}+\mathrm{H})^{+}, 326(7), 207(100), 164(6), 120$ (12).

Allene 23

$\mathrm{R}_{\mathrm{f}}=0.43\left[25 \%\right.$ EtOAc in hexanes]; $\mathrm{mp}=95-97^{\circ} \mathrm{C} ;[\alpha]_{\mathrm{D}}{ }^{23}-8.7\left(\mathrm{c} 0.60, \mathrm{CH}_{2} \mathrm{Cl}_{2}\right) ;{ }^{\prime} \mathrm{H} \mathrm{NMR}\left(500 \mathrm{MHz}, \mathrm{CDCl}_{3}\right) \delta$ 0.86-1.02 (m, $5 \mathrm{H}), 1.06-1.20(\mathrm{~m}, 2 \mathrm{H}), 1.24-1.36(\mathrm{~m}, 4 \mathrm{H}), 1.49-1.63(\mathrm{~m}, 6 \mathrm{H}), 1.70-1.88(\mathrm{~m}, 3 \mathrm{H}), 4.25$ (dd, 1 $\mathrm{H}, J=4.0,9.0 \mathrm{~Hz}), 4.27(\mathrm{dq}, 1 \mathrm{H}, J=2.0,6.5 \mathrm{~Hz}), 4.69(\mathrm{t}, 1 \mathrm{H}, J=9.0 \mathrm{~Hz}), 5.20(\mathrm{dt}, 1 \mathrm{H}, J=4.0,6.0 \mathrm{~Hz}), 5.23$ $(\mathrm{dt}, 1 \mathrm{H}, J=4.0,6.0 \mathrm{~Hz}), 5.45(\mathrm{dd}, 1 \mathrm{H}, J=4.0,9.0 \mathrm{~Hz}), 7.26-7.40(\mathrm{~m}, 5 \mathrm{H}) ;{ }^{13} \mathrm{C} \mathrm{NMR}\left(75 \mathrm{MHz}, \mathrm{CDCl}_{3}\right) \delta$ $14.0,22.6,26.0,26.1,29.4,30.5,32.8,32.9,37.0,37.2,43.2,57.7,69.8,90.0,99.5,125.9,128.6,129.1,138.9$, 153.4, 173.5, 203.7; IR (thin film) $\mathrm{cm}^{-1} 2924(\mathrm{~m}), 2859(\mathrm{w}), 1785(\mathrm{~s}), 1707(\mathrm{~s}), 1457(\mathrm{~m}), 1379$ (m); mass spectrum (APCI): $\mathrm{m} / \mathrm{e}$ (\% relative intensity) $382(66)(\mathrm{M}+\mathrm{H})^{+}, 219(100), 191(6), 164(8), 120(9)$. 
(C) 2003 American Chemical Society, Org. Lett., Frederick ol030061c Supporting Info Page 4

Allene 24

$\mathrm{R}_{\mathrm{f}}=0.38\left[25 \%\right.$ EtOAc in hexanes]; clear oil; $[\alpha]_{\mathrm{D}}{ }^{23}+55.6\left(\mathrm{c} 0.63, \mathrm{CH}_{2} \mathrm{Cl}_{2}\right) ;{ }^{1} \mathrm{H} \mathrm{NMR}\left(500 \mathrm{MHz}, \mathrm{CDCl}_{3}\right) \delta$ 0.84-1.40 (m, $6 \mathrm{H}), 1.54$ (dd, $3 \mathrm{H}, J=3.0,7.0 \mathrm{~Hz}), 1.64-1.76(\mathrm{~m}, 4 \mathrm{H}), 1.86(\mathrm{~d}, 1 \mathrm{H}, J=7.0 \mathrm{~Hz}), 4.24(\mathrm{dd}, 1 \mathrm{H}$, $J=4.0,8.5 \mathrm{~Hz}), 4.25(\mathrm{t}, 1 \mathrm{H}, J=9.0 \mathrm{~Hz}), 4.68(\mathrm{t}, 1 \mathrm{H}, J=8.5 \mathrm{~Hz}), 4.97$ (septet, $1 \mathrm{H}, J=3.0 \mathrm{~Hz}$ ), 5.09 (quintet, $1 \mathrm{H}, J=7.0 \mathrm{~Hz}), 5.45(\mathrm{dd}, 1 \mathrm{H}, J=4.0,9.0 \mathrm{~Hz}), 7.27-7.39(\mathrm{~m}, 5 \mathrm{H}) ;{ }^{13} \mathrm{C} \mathrm{NMR}\left(75 \mathrm{MHz} \mathrm{CDCl}_{3}\right) \delta 13.9,26.0$, 26.2, 29.5, 31.2, 38.3, 39.7, 49.5, 57.6, 69.5, 86.6, 87.4, 125.7, 128.4, 128.9, 138.8, 153.1, 173.2, 205.8; IR (thin film) $\mathrm{cm}^{-1} 2924(\mathrm{~m}), 2854(\mathrm{w}), 1783(\mathrm{~s}), 1700(\mathrm{~m}), 1386(\mathrm{~m})$; mass spectrum (APCI): m/e (\% relative intensity) $340(48)(\mathrm{M}+\mathrm{H})^{+}, 177(100), 164(14), 149(87), 87(62)$.

Allene 25

$\mathrm{R}_{\mathrm{f}}=0.27$ [25\% EtOAc in hexanes]; clear oil; $[\alpha]_{\mathrm{D}}{ }^{23}-88.7\left(\mathrm{c} 0.37, \mathrm{CH}_{2} \mathrm{Cl}_{2}\right) ;{ }^{1} \mathrm{H} \mathrm{NMR}\left(500 \mathrm{MHz}, \mathrm{CDCl}_{3}\right) \delta 1.23$ $(\mathrm{d}, 3 \mathrm{H}, J=7.0 \mathrm{~Hz}$ ), 1.52 (dd, $3 \mathrm{H}, J=3.0,7.0 \mathrm{~Hz}$ ), 4.27 (dd, $1 \mathrm{H}, J=4.5,9.0 \mathrm{~Hz}$ ), 4.33 (ddt, $1 \mathrm{H}, J=2.5,7.0$, $7.0 \mathrm{~Hz}), 4.70(\mathrm{t}, 1 \mathrm{H}, J=9.0 \mathrm{~Hz}), 5.17(\mathrm{ddt}, 1 \mathrm{H}, J=2.5,7.0,7.0 \mathrm{~Hz}), 5.29(\mathrm{ddd}, 1 \mathrm{H}, J=3.0,7.0,7.0 \mathrm{~Hz}), 5.45$ $(\mathrm{dd}, 1 \mathrm{H}, J=4.5,9.0 \mathrm{~Hz}), 7.32-7.34(\mathrm{~m}, 3 \mathrm{H}), 7.35-7.40(\mathrm{~m}, 2 \mathrm{H}) ;{ }^{13} \mathrm{C} \mathrm{NMR}\left(75 \mathrm{MHz}, \mathrm{CDCl}_{3}\right) \delta 13.9,15.9$, $37.4,57.6,69.7,88.5,89.9,125.8,128.5,128.9,129.0,153.1,173.7,204.7$; IR (thin film) $\mathrm{cm}^{-1} 2955(\mathrm{~m}), 2940$ $(\mathrm{m}), 2870(\mathrm{w}), 1776(\mathrm{~s}), 1703(\mathrm{~s})$; mass spectrum (APCI): m/e (\% relative intensity) $272(15)(\mathrm{M}+\mathrm{H})^{+}, 228$ (14), $164(19), 120(54), 109(100)$.

\section{Allene 26}

$\mathrm{R}_{\mathrm{f}}=0.24$ [25\% EtOAc in hexanes]; clear oil; $[\alpha]_{\mathrm{D}}^{23}+7.1\left(\mathrm{c} 0.34, \mathrm{CH}_{2} \mathrm{Cl}_{2}\right) ;{ }^{2} \mathrm{H} \mathrm{NMR}\left(500 \mathrm{MHz}, \mathrm{CDCl}_{3}\right) \delta 1.31$ $(\mathrm{d}, 3 \mathrm{H}, J=7.0 \mathrm{~Hz}$ ), 4.26 (dd, $1 \mathrm{H}, J=4.5,9.0 \mathrm{~Hz}$ ), 4.51 (ddt, $1 \mathrm{H}, J=3.0,7.0,7.0 \mathrm{~Hz}), 4.71(\mathrm{t}, 1 \mathrm{H}, J=9.0$ $\mathrm{Hz}$ ), $5.46(\mathrm{dd}, 1 \mathrm{H}, J=4.5,9.0 \mathrm{~Hz}), 5.83(\mathrm{t}, 1 \mathrm{H}, J=7.0 \mathrm{~Hz}), 6.22(\mathrm{dd}, 1 \mathrm{H}, J=3.0,7.0 \mathrm{~Hz}), 7.12-7.40(\mathrm{~m}, 10$ $\mathrm{H}) ;{ }^{13} \mathrm{C} \mathrm{NMR}\left(75 \mathrm{MHz} \mathrm{CDCl}_{3}\right.$ ) $\delta 15.8,37.8,57.8,69.8,94.8,97.4,125.8,126.7,126.9,128.4,128.5,129.0$, 133.7, 138.5, 153.4, 173.2, 203.2; IR (thin film) $\mathrm{cm}^{-1} 2963(\mathrm{~m}), 2935(\mathrm{~m}), 2866(\mathrm{w}), 1781(\mathrm{~s}), 1706(\mathrm{~s}), 1391$ (m); mass spectrum (APCI): m/e (\% relative intensity) $334(13)(\mathrm{M}+\mathrm{H})^{+}, 261(54), 247(38), 229(43), 171$ (100), 147 (36), $115(35)$.

\section{Allene 27}

$\mathrm{R}_{\mathrm{f}}=0.41\left[25 \%\right.$ EtOAc in hexanes]; clear oil; $[\alpha]_{\mathrm{D}}{ }^{23}-30.9\left(\mathrm{c} 0.59, \mathrm{CH}_{2} \mathrm{Cl}_{2}\right) ;{ }^{1} \mathrm{H} \mathrm{NMR}\left(500 \mathrm{MHz}, \mathrm{CDCl}_{3}\right) \delta 0.88$ $(\mathrm{t}, 3 \mathrm{H}, J=7.0 \mathrm{~Hz}), 1.28-1.39(\mathrm{~m}, 8 \mathrm{H}), 1.58-1.62(\mathrm{~m}, 1 \mathrm{H}), 1.68(\mathrm{dd}, 3 \mathrm{H}, J=3.0,7.0 \mathrm{~Hz}), 1.79-1.83(\mathrm{~m}, 1 \mathrm{H})$, $2.73(\mathrm{dd}, 1 \mathrm{H}, J=10.0,13.5 \mathrm{~Hz}), 3.30(\mathrm{dd}, 1 \mathrm{H}, J=3.5,13.5 \mathrm{~Hz}), 4.16(\mathrm{dd}, 1 \mathrm{H}, J=3.0,7.0 \mathrm{~Hz}), 4.20(\mathrm{t}, 1 \mathrm{H}, J$ $=9.0 \mathrm{~Hz}), 4.27(\mathrm{dq}, 1 \mathrm{H}, J=2.0,7.0 \mathrm{~Hz}), 4.66-4.71(\mathrm{~m}, 1 \mathrm{H}), 5.22(\mathrm{dq}, 1 \mathrm{H}, J=2.0,7.0 \mathrm{~Hz}), 5.28-5.32(\mathrm{~m}, 1$ $\mathrm{H}), 7.22-7.35(\mathrm{~m}, 5 \mathrm{H}) ;{ }^{13} \mathrm{C} \mathrm{NMR}\left(75 \mathrm{MHz}, \mathrm{CDCl}_{3}\right) \delta 14.0,14.2,22.5,27.0,29.0,31.6,31.7,37.6,43.3,55.2$, $65.8,87.7,89.2,127.1,128.8,129.3,135.2,152.7,174.0,204.9$; IR (thin film) $\mathrm{cm}^{-1} 2952(\mathrm{~m}), 2930(\mathrm{~m}), 2865$ $(\mathrm{w}), 1786(\mathrm{~s}), 1706(\mathrm{~s}), 1460(\mathrm{~m})$; mass spectrum $(\mathrm{APCI}): \mathrm{m} / \mathrm{e}$ (\% relative intensity) $356(43)(\mathrm{M}+\mathrm{H})^{+}, 330$ (25), $312(12), 179(100), 117(22)$.

\section{Allene 28}

$\mathrm{R}_{\mathrm{f}}=0.42\left[25 \%\right.$ EtOAc in hexanes]; $\mathrm{mp}=152-154^{\circ} \mathrm{C} ;[\alpha]_{\mathrm{D}}{ }^{23}+27.2\left(\mathrm{c} 0.34, \mathrm{CH}_{2} \mathrm{Cl}_{2}\right) ;{ }^{\prime} \mathrm{H}$ NMR $(500 \mathrm{MHz}$, $\left.\mathrm{CDCl}_{3}\right) \delta 0.87(\mathrm{t}, 3 \mathrm{H}, J=7.0 \mathrm{~Hz}), 1.25-1.36(\mathrm{~m}, 4 \mathrm{H}), 1.61-1.68(\mathrm{~m}, 1 \mathrm{H}), 1.77-1.84(\mathrm{~m}, 1 \mathrm{H}), 4.38-4.44(\mathrm{~m}, 2$ $\mathrm{H}), 4.45(\mathrm{t}, 1 \mathrm{H}, J=9.0 \mathrm{~Hz}), 4.77(\mathrm{~d}, 1 \mathrm{H}, J=6.0 \mathrm{~Hz}), 5.38(\mathrm{ddd}, 1 \mathrm{H}, J=3.5,6.0,7.5 \mathrm{~Hz}), 5.48(\mathrm{dd}, 1 \mathrm{H}, J=$ $7.0,8.0 \mathrm{~Hz}), 6.28(\mathrm{dd}, 1 \mathrm{H}, J=2.0,6.5), 7.14-7.35(\mathrm{~m}, 15 \mathrm{H}) ;{ }^{13} \mathrm{C} \mathrm{NMR}\left(75 \mathrm{MHz}, \mathrm{CDCl}_{3}\right) \delta 13.9,22.4,29.4$, $31.0,43.5,50.7,56.3,64.7,93.7,96.3,126.5,126.8,127.0,127.8,128.3,128.5,128.6,128.8,129.3,134.1$, 137.8, 139.4, 152.8, 173.7, 205.8; IR (thin film) $\mathrm{cm}^{-1} 3031$ (w), 2957 (m), 2930 (m), $2870(\mathrm{w}), 1784$ (s), 1700 (s); mass spectrum (APCI): $\mathrm{m} / \mathrm{e}$ (\% relative intensity) $466(71)(\mathrm{M}+\mathrm{H})^{+}, 276(8), 254$ (22), 213 (100), 193 (13).

Allene 30

$\mathrm{R}_{\mathrm{f}}=0.71$ (40\% EtOAc in hexanes); oil; $[\alpha]_{\mathrm{D}}{ }^{23}-36.7$ (c $\left.0.22, \mathrm{EtOH}\right) ;{ }^{1} \mathrm{H}$ NMR $\left(500 \mathrm{MHz}, \mathrm{CDCl}_{3}\right.$ ) major/minor mostly overlapped $\delta 0.88(\mathrm{t}, J=6.5 \mathrm{~Hz}, 3 \mathrm{H}), 0.91(\mathrm{~s}, 3 \mathrm{H}), 1.02(\mathrm{~s}, 3 \mathrm{H}), 1.26-1.36(\mathrm{~m}, 4 \mathrm{H}), 1.45(\mathrm{~s}, 3 \mathrm{H}), 1.47$ $(\mathrm{s}, 1 \mathrm{H}), 1.64(\mathrm{~m}, 1 \mathrm{H}), 1.63(\mathrm{dd}, J=3.3,6.8 \mathrm{~Hz}, 3 \mathrm{H}) 1.72-1.85(\mathrm{~m}, 2 \mathrm{H}), 1.96-2.10(\mathrm{~m}, 2 \mathrm{H}), 2.36(\mathrm{~d}, J=4.0 \mathrm{~Hz}$, 
(C) 2003 American Chemical Society, Org. Lett., Frederick ol030061c Supporting Info Page 5

$1 \mathrm{H}), 4.17(\mathrm{~m}, 1 \mathrm{H}), 5.10-5.20(\mathrm{~m}, 2 \mathrm{H}) ;{ }^{13} \mathrm{C} \mathrm{NMR}\left(75 \mathrm{MHz}, \mathrm{CDCl}_{3}\right)$ major only $\delta 13.1,13.5,13.9,14.2,14.3$, $17.5,18.5,22.5,23.6,29.1,30.3,32.0,46.4,55.3,87.1,90.0,176.0,177.7,205.0$; IR (thin film) $\mathrm{cm}^{-1} 2957 \mathrm{~m}$, $2927 \mathrm{~m}, 1743 \mathrm{~s}, 1699 \mathrm{~s}$; mass spectrum [EI]; m/e (\%relative intensity) 303 (1) $\mathrm{M}^{+}, 246$ (43), 220 (47), $166(56)$, $136(45), 110(100)$.

Allene 31

$\mathrm{R}_{\mathrm{f}}=0.71$ (40\% EtOAc in hexanes); oil; $[\alpha]_{\mathrm{D}}{ }^{23}-89.4$ (c $\left.0.26, \mathrm{EtOH}\right) ;{ }^{\prime} \mathrm{H} \mathrm{NMR}\left(300 \mathrm{MHz}, \mathrm{CDCl}_{3}\right.$ ) major/minor mostly overlapped $\delta 0.89(\mathrm{t}, J=3.3 \mathrm{~Hz}, 3 \mathrm{H}), 0.91(\mathrm{~s}, 3 \mathrm{H}), 1.02(\mathrm{~s}, 3 \mathrm{H}), 1.27-1.42(\mathrm{~m}, 4 \mathrm{H}), 1.44(\mathrm{~s}, 3 \mathrm{H}), 1.48(\mathrm{~s}$, $1 \mathrm{H}), 1.62-1.66(\mathrm{~m}, 4 \mathrm{H}), 1.72-1.87(\mathrm{~m}, 2 \mathrm{H}), 1.94-2.10(\mathrm{~m}, 2 \mathrm{H}), 2.36(\mathrm{~d}, J=4.2 \mathrm{~Hz}, 1 \mathrm{H}), 4.18(\mathrm{~m}, 1 \mathrm{H}), 5.12-5.15$ $(\mathrm{m}, 2 \mathrm{H}) ;{ }^{13} \mathrm{C}$ NMR $\left(125 \mathrm{MHz}, \mathrm{CDCl}_{3}\right.$ ) major/minor $\delta 13.5,13.7,13.8,14.2,14.5,14.6,17.8,17.9,18.9,19.4$, $22.7,23.9,24.0,24.5,26.1,29.4,29.5,30.6,32.3,32.4,32.8,39.1,46.7,47.7,55.5,55.6,73.3,87.1,87.4,89.1$, $90.4,112.5,176.4,178.1,205.4$; IR (thin film) $\mathrm{cm}^{-1} 2960 \mathrm{~m}, 2932 \mathrm{~m}, 1743 \mathrm{~s}, 1699 \mathrm{~s}$; mass spectrum [EI]: $\mathrm{m} / \mathrm{e}$ (\%relative intensity) 303 (1) $\mathrm{M}^{+}, 246(60), 220(60), 166(62), 136(50), 110(100)$.

Allene 32

$\mathrm{R}_{\mathrm{f}}=0.25\left[25 \%\right.$ EtOAc in hexanes]; $\mathrm{mp}=84-86^{\circ} \mathrm{C} ;[\alpha]_{\mathrm{D}}^{23}+98.7\left(\mathrm{c} 0.46, \mathrm{CH}_{2} \mathrm{Cl}_{2}\right) ;{ }^{1} \mathrm{H} \mathrm{NMR}^{2}\left(500 \mathrm{MHz}, \mathrm{CDCl}_{3}\right)$ $\delta 0.88(\mathrm{t}, 3 \mathrm{H}, J=7.0 \mathrm{~Hz}), 1.29-1.40(\mathrm{~m}, 4 \mathrm{H}), 1.61-1.64(\mathrm{~m}, 1 \mathrm{H}), 1.83-1.85(\mathrm{~m}, 1 \mathrm{H}), 4.25(\mathrm{dd}, 1 \mathrm{H}, J=4.0$, $9.0 \mathrm{~Hz}), 4.45(\mathrm{dq}, 1 \mathrm{H}, J=2.0,7.0 \mathrm{~Hz}), 4.70(\mathrm{t}, 1 \mathrm{H}, J=9.0 \mathrm{~Hz}), 5.45(\mathrm{dd}, 1 \mathrm{H}, J=4.0,9.0 \mathrm{~Hz}), 5.70(\mathrm{t}, 1 \mathrm{H}, J$ $=7.0 \mathrm{~Hz}), 6.19(\mathrm{dd}, 1 \mathrm{H}, J=2.0,7.0 \mathrm{~Hz}), 7.13-7.32(\mathrm{~m}, 10 \mathrm{H}) ;{ }^{13} \mathrm{C} \mathrm{NMR}\left(75 \mathrm{MHz}, \mathrm{CDCl}_{3}\right) \delta 14.1,22.6,29.6$, $30.8,43.5,57.9,69.9,93.7,97.0,126.0,126.7,126.9,128.6,128.7,129.1,134.0,140.2,153.4,173.2,205.6$; IR (thin film) $\mathrm{cm}^{-1} 2964(\mathrm{~m}), 2933(\mathrm{~m}), 2865(\mathrm{w}), 1781(\mathrm{~s}), 1707(\mathrm{~m}), 1385(\mathrm{~m})$; mass spectrum (APCI): m/e (\% relative intensity) $376(20)(\mathrm{M}+\mathrm{H})^{+}, 332(8), 213(100), 185(16), 120(7)$.

Allene 33

$\mathrm{R}_{\mathrm{r}}=0.25\left[25 \%\right.$ EtOAc in hexanes]; $\mathrm{mp}=95-97^{\circ} \mathrm{C} ;[\alpha]_{\mathrm{D}}{ }^{23}-150.8\left(\mathrm{c} 0.39, \mathrm{CH}_{2} \mathrm{Cl}_{2}\right) ;{ }^{\prime} \mathrm{H}$ NMR $\left(500 \mathrm{MHz}, \mathrm{CDCl}_{3}\right)$ $\delta 0.88(\mathrm{t}, 3 \mathrm{H}, J=7.0 \mathrm{~Hz}), 1.26-1.40(\mathrm{~m}, 4 \mathrm{H}), 1.58-1.65(\mathrm{~m}, 1 \mathrm{H}), 1.83-1.89(\mathrm{~m}, 1 \mathrm{H}), 4.29(\mathrm{dd}, 1 \mathrm{H}, J=4.0$, $9.0 \mathrm{~Hz}), 4.42(\mathrm{dq}, 1 \mathrm{H}, J=2.0,7.0 \mathrm{~Hz}), 4.70(\mathrm{t}, 1 \mathrm{H}, J=9.0 \mathrm{~Hz}), 5.47(\mathrm{dd}, 1 \mathrm{H}, J=4.0,8.5 \mathrm{~Hz}), 5.73(\mathrm{t}, 1 \mathrm{H}, J$ $=7.0 \mathrm{~Hz}), 6.00(\mathrm{dd}, 1 \mathrm{H}, J=2.0,7.0 \mathrm{~Hz}), 7.15-7.56(\mathrm{~m}, 10 \mathrm{H}) ;{ }^{13} \mathrm{C} \mathrm{NMR}\left(75 \mathrm{MHz}, \mathrm{CDCl}_{3}\right) \delta 14.0,22.6,29.5$, $31.3,43.5,57.7,69.8,93.6,96.8,126.0,126.6,126.8,128.6,128.7,129.2,133.8,138.8,153.3,172.8,205.5$; IR (thin film) $\mathrm{cm}^{-1} 2964(\mathrm{~m}), 2933(\mathrm{~m}), 2865(\mathrm{w}), 1781(\mathrm{~s}), 1707(\mathrm{~m}), 1385(\mathrm{~m})$; mass spectrum (APCI): m/e (\% relative intensity) $376(20)(\mathrm{M}+\mathrm{H})^{+}, 332(8), 213(100), 185(16), 120(7)$.

Allene 34

$\mathrm{R}_{\mathrm{f}}=0.25\left[25 \%\right.$ EtOAc in hexanes]; $\mathrm{mp}=86-87^{\circ} \mathrm{C} ;[\alpha]_{\mathrm{D}}{ }^{23}-99.1\left(\mathrm{c} 0.54, \mathrm{CH}_{2} \mathrm{Cl}_{2}\right) ;{ }^{1} \mathrm{H}$ NMR $\left(500 \mathrm{MHz}, \mathrm{CDCl}_{3}\right)$ $\delta 0.88(\mathrm{t}, 3 \mathrm{H}, J=7.0 \mathrm{~Hz}), 1.29-1.40(\mathrm{~m}, 4 \mathrm{H}), 1.61-1.64(\mathrm{~m}, 1 \mathrm{H}), 1.83-1.85(\mathrm{~m}, 1 \mathrm{H}), 4.25(\mathrm{dd}, 1 \mathrm{H}, J=4.0$, $9.0 \mathrm{~Hz}), 4.45(\mathrm{dq}, 1 \mathrm{H}, J=2.0,7.0 \mathrm{~Hz}), 4.70(\mathrm{t}, 1 \mathrm{H}, J=9.0 \mathrm{~Hz}), 5.45(\mathrm{dd}, 1 \mathrm{H}, J=4.0,9.0 \mathrm{~Hz}), 5.70(\mathrm{t}, 1 \mathrm{H}, J$ $=7.0 \mathrm{~Hz}), 6.19(\mathrm{dd}, 1 \mathrm{H}, J=2.0,7.0 \mathrm{~Hz}), 7.13-7.32(\mathrm{~m}, 10 \mathrm{H}) ;{ }^{13} \mathrm{C} \mathrm{NMR}\left(75 \mathrm{MHz}, \mathrm{CDCl}_{3}\right) \delta 14.1,22.6,29.6$, $30.8,43.5,57.9,69.9,93.7,97.0,126.0,126.7,126.9,128.6,128.7,129.1,134.0,140.2,153.4,173.2,205.6$; IR (thin film) $\mathrm{cm}^{-1} 2964(\mathrm{~m}), 2933(\mathrm{~m}), 2865(\mathrm{w}), 1781(\mathrm{~s}), 1707(\mathrm{~m}), 1385(\mathrm{~m}) ;$ mass spectrum (APCI): $\mathrm{m} / \mathrm{e}(\%$ relative intensity) $376(20)(\mathrm{M}+\mathrm{H})^{+}, 332(8), 213(100), 185(16), 120(7)$.

Allene 35

$\mathrm{R}_{\mathrm{f}}=0.25$ [25\% EtOAc in hexanes]; $\mathrm{mp}=81-83^{\circ} \mathrm{C} ;[\alpha]_{\mathrm{D}}{ }^{23}+126.5\left(\mathrm{c} 0.80, \mathrm{CH}_{2} \mathrm{Cl}_{2}\right) ;{ }^{\prime} \mathrm{H}$ NMR $\left(500 \mathrm{MHz}, \mathrm{CDCl}_{3}\right)$ $\delta 0.88(\mathrm{t}, 3 \mathrm{H}, J=7.0 \mathrm{~Hz}), 1.26-1.40(\mathrm{~m}, 4 \mathrm{H}), 1.58-1.65(\mathrm{~m}, 1 \mathrm{H}), 1.83-1.89(\mathrm{~m}, 1 \mathrm{H}), 4.29(\mathrm{dd}, 1 \mathrm{H}, J=4.0$, $9.0 \mathrm{~Hz}), 4.42(\mathrm{dq}, 1 \mathrm{H}, J=2.0,7.0 \mathrm{~Hz}), 4.70(\mathrm{t}, 1 \mathrm{H}, J=9.0 \mathrm{~Hz}), 5.47(\mathrm{dd}, 1 \mathrm{H}, J=4.0,8.5 \mathrm{~Hz}), 5.73(\mathrm{t}, 1 \mathrm{H}, J$ $=7.0 \mathrm{~Hz}), 6.00(\mathrm{dd}, 1 \mathrm{H}, J=2.0,7.0 \mathrm{~Hz}), 7.15-7.56(\mathrm{~m}, 10 \mathrm{H}) ;{ }^{13} \mathrm{C} \mathrm{NMR}\left(75 \mathrm{MHz}, \mathrm{CDCl}_{3}\right) \delta 14.0,22.6,29.5$, $31.3,43.5,57.7,69.8,93.6,96.8,126.0,126.6,126.8,128.6,128.7,129.2,133.8,138.8,153.3,172.8,205.5 ;$ IR (thin film) $\mathrm{cm}^{-1} 2964(\mathrm{~m}), 2933(\mathrm{~m}), 2865(\mathrm{w}), 1781(\mathrm{~s}), 1707(\mathrm{~m}), 1385(\mathrm{~m})$; mass spectrum (APCI): $\mathrm{m} / \mathrm{e}(\%$ relative intensity) $376(20)(\mathrm{M}+\mathrm{H})^{+}, 332(8), 213(100), 185(16), 120(7)$. 
(C) 2003 American Chemical Society, Org. Lett., Frederick ol030061c Supporting Info Page 6

Allene 38

$\mathrm{R}_{\mathrm{f}}=0.50$ [25\% EtOAc in hexanes]; clear oil; $[\alpha]_{\mathrm{D}}{ }^{23}-94.0\left(\mathrm{c} 0.90, \mathrm{CH}_{2} \mathrm{Cl}_{2}\right) ;{ }^{\prime} \mathrm{H} \mathrm{NMR}\left(500 \mathrm{MHz}, \mathrm{CDCl}_{3}\right) \delta 0.86$ $(\mathrm{t}, 3 \mathrm{H}, J=7.0 \mathrm{~Hz}), 0.92(\mathrm{t}, 3 \mathrm{H}, J=7.0 \mathrm{~Hz}), 1.24-1.28(\mathrm{~m}, 2 \mathrm{H}), 1.32-1.42(\mathrm{~m}, 6 \mathrm{H}), 1.52-1.55(\mathrm{~m}, 2 \mathrm{H}), 1.71-$ $1.92(\mathrm{~m}, 4 \mathrm{H}), 4.18(\mathrm{dd}, 1 \mathrm{H}, J=4.5,9.0 \mathrm{~Hz}), 4.65(\mathrm{t}, 1 \mathrm{H}, J=9.0 \mathrm{~Hz}), 5.05(\mathrm{dt}, 1 \mathrm{H}, J=2.0,7.0 \mathrm{~Hz}), 5.44(\mathrm{dd}$, $1 \mathrm{H}, J=4.5,9.0 \mathrm{~Hz}), 5.53(\mathrm{dt}, 1 \mathrm{H}, J=2.0,7.0), 7.18-7.45(\mathrm{~m}, 10 \mathrm{H}) ;{ }^{13} \mathrm{C} \mathrm{NMR}\left(75 \mathrm{MHz}, \mathrm{CDCl}_{3}\right) \delta 13.9,22.3$, $22.6,28.4,29.0,29.8,31.3,31.6,43.5,57.8,65.5,69.5,90.1,96.5,125.7,126.3,128.1,128.2,128.8,131.5$, 136.5, 138.4, 153.4, 173.3, 204.5; IR (thin film) $\mathrm{cm}^{-1} 2960(\mathrm{~m}), 2932(\mathrm{~m}), 2862(\mathrm{w}), 1784(\mathrm{~s}), 1702$ (s); mass spectrum (APCI): $\mathrm{m} / \mathrm{e}$ (\% relative intensity) $446(8)(\mathrm{M}+\mathrm{H})^{+}, 284(22), 283(100)$.

\section{Allene 39}

$\mathrm{R}_{\mathrm{f}}=0.48\left[25 \%\right.$ EtOAc in hexanes]; clear oil; $[\alpha]_{\mathrm{D}}{ }^{23}-47.5\left(\mathrm{c} 0.40, \mathrm{CH}_{2} \mathrm{Cl}_{2}\right) ;{ }^{1} \mathrm{H} \mathrm{NMR}\left(500 \mathrm{MHz}, \mathrm{CDCl}_{3}\right) \delta 0.82$ $(\mathrm{t}, 3 \mathrm{H}, J=7.0 \mathrm{~Hz}), 0.92(\mathrm{t}, 3 \mathrm{H}, J=7.0 \mathrm{~Hz}), 0.94-0.97(\mathrm{~m}, 2 \mathrm{H}), 1.07-1.32(\mathrm{~m}, 12 \mathrm{H}), 1.55-1.80(\mathrm{~m}, 9 \mathrm{H}), 4.24$ $(\mathrm{dd}, 1 \mathrm{H}, J=4.5,8.5 \mathrm{~Hz}), 4.41(\mathrm{t}, 1 \mathrm{H}, J=7.0 \mathrm{~Hz}), 4.67(\mathrm{t}, 1 \mathrm{H}, J=8.5 \mathrm{~Hz}), 5.16-5.18(\mathrm{~m}, 1 \mathrm{H}), 5.46(\mathrm{dd}, 1 \mathrm{H}, J$ $=4.5,8.5 \mathrm{~Hz}), 7.28-7.36(\mathrm{~m}, 5 \mathrm{H}) ;{ }^{13} \mathrm{C}$ NMR $\left(75 \mathrm{MHz}, \mathrm{CDCl}_{3}\right) \delta 13.9,18.2,22.2,22.5,25.8,26.0,27.9,29.5$, 29.6, 30.2, 30.7, 33.0, 37.5, 44.2, 46.2, 57.7, 67.3, 100.1, 103.1, 125.9, 128.4, 128.8, 138.8, 153.3, 173.6, 200.9; IR (thin film) $\mathrm{cm}^{-1} 2958(\mathrm{~m}), 2940(\mathrm{~m}), 2856(\mathrm{w}), 1784(\mathrm{~s}), 1701(\mathrm{~s}) ;$ mass spectrum (APCI): m/e (\% relative intensity) $438(73)(\mathrm{M}+\mathrm{H})^{+}, 394(7), 340(10), 275$ (100), 177 (17).

\section{Allene 40}

$\mathrm{R}_{\mathrm{f}}=0.39$ [25\% EtOAc in hexanes]; clear oil; ' ${ }^{1} \mathrm{H}$ NMR (500 MHz, $\left.\mathrm{CDCl}_{3}\right)$ Major isomer: $\delta 0.81-0.91(\mathrm{~m}, 6 \mathrm{H})$, $1.21-1.57(\mathrm{~m}, 11 \mathrm{H}), 1.70-2.01(\mathrm{~m}, 3 \mathrm{H}), 4.26(\mathrm{dd}, 1 \mathrm{H}, J=4.2,9.0 \mathrm{~Hz}), 4.31-4.36(\mathrm{~m}, 1 \mathrm{H}), 4.68(\mathrm{t}, 1 \mathrm{H}, J=9.0$ $\mathrm{Hz}), 5.19-5.25(\mathrm{~m}, 2 \mathrm{H}), 5.45(\mathrm{t}, 1 \mathrm{H}, J=9.0 \mathrm{~Hz}), 7.27-7.40(\mathrm{~m}, 5 \mathrm{H})$; Minor isomer: $\delta 0.81-0.91(\mathrm{~m}, 6 \mathrm{H}), 1.21-$ $1.57(\mathrm{~m}, 11 \mathrm{H}), 1.70-2.01(\mathrm{~m}, 3 \mathrm{H}), 4.28(\mathrm{dd}, 1 \mathrm{H}, J=4.2,9.0 \mathrm{~Hz}), 4.31-4.36(\mathrm{~m}, 1 \mathrm{H}), 4.70(\mathrm{t}, 1 \mathrm{H}, J=9.0 \mathrm{~Hz})$, $4.98(\mathrm{dq}, 1 \mathrm{H}, J=1.8,6.6 \mathrm{~Hz}), 5.20-5.25(\mathrm{~m}, 1 \mathrm{H}), 5.44(\mathrm{t}, 1 \mathrm{H}, J=9.0 \mathrm{~Hz}), 7.27-7.40(\mathrm{~m}, 5 \mathrm{H})$; IR (thin film) $\mathrm{cm}^{-1} 2965(\mathrm{~m}), 2930(\mathrm{~m}), 2925(\mathrm{~m}), 2860(\mathrm{w}), 1777(\mathrm{~s}), 1702(\mathrm{~s}) ;$ mass spectrum (APCI): $\mathrm{m} / \mathrm{e}$ (\% relative intensity) $370(46)(\mathrm{M}+\mathrm{H})^{+}, 326(9), 207(100), 120(26), 87$ (12).

Hydrogenated Product 41

$\mathrm{R}_{\mathrm{f}}=0.39$ [25\% EtOAc in hexanes]; clear oil; ' $\mathrm{H}$ NMR (500 MHz, $\mathrm{CDCl}_{3}$ ) Major isomer: $\delta 0.77(\mathrm{t}, 3 \mathrm{H}, J=6.9$ $\mathrm{Hz}), 0.85-0.91(\mathrm{~m}, 3 \mathrm{H}), 0.88(\mathrm{t}, 3 \mathrm{H}, J=6.9 \mathrm{~Hz}), 0.98-1.08(\mathrm{~m}, 4 \mathrm{H}), 1.14-1.29(\mathrm{~m}, 8 \mathrm{H}), 1.36-1.46(\mathrm{~m}, 3 \mathrm{H})$, 1.51-1.67 (m, $2 \mathrm{H}), 3.77-3.86(\mathrm{~m}, 1 \mathrm{H}), 4.26(\mathrm{dd}, 1 \mathrm{H}, J=3.9,9.0 \mathrm{~Hz}), 4.68(\mathrm{t}, 1 \mathrm{H}, J=9.0 \mathrm{~Hz}), 5.46(\mathrm{dd}, 1 \mathrm{H}$, $J=3.9,9.0 \mathrm{~Hz}), 7.27-7.40(\mathrm{~m}, 5 \mathrm{H})$; Minor isomer: $\delta 0.77(\mathrm{t}, 3 \mathrm{H}, J=6.9 \mathrm{~Hz}), 0.85-0.91(\mathrm{~m}, 3 \mathrm{H}), 0.88(\mathrm{t}, 3 \mathrm{H}$, $J=6.9 \mathrm{~Hz}), 0.98-1.08(\mathrm{~m}, 4 \mathrm{H}), 1.14-1.29(\mathrm{~m}, 8 \mathrm{H}), 1.36-1.46(\mathrm{~m}, 3 \mathrm{H}), 1.51-1.67(\mathrm{~m}, 2 \mathrm{H}), 3.77-3.86(\mathrm{~m}, 1 \mathrm{H})$, $4.27(\mathrm{dd}, 1 \mathrm{H}, J=3.9,9.0 \mathrm{~Hz}), 4.68(\mathrm{t}, 1 \mathrm{H}, J=9.0 \mathrm{~Hz}), 5.46(\mathrm{dd}, 1 \mathrm{H}, J=3.9,9.0 \mathrm{~Hz}), 7.27-7.40(\mathrm{~m}, 5 \mathrm{H}) ; \mathrm{IR}$ (thin film) $\mathrm{cm}^{-1} 2955(\mathrm{~m}), 2942(\mathrm{~m}), 2862(\mathrm{w}), 1782(\mathrm{~s}), 1703(\mathrm{~s})$; mass spectrum (APCI): $\mathrm{m} / \mathrm{e}$ (\% relative intensity) $374(100)(\mathrm{M}+\mathrm{H})^{+}, 282(22), 211(22), 183(75), 164(82), 120$ (23).

Hydrogenated Product 42

$\mathrm{R}_{\mathrm{f}}=0.25$ [25\% EtOAc in hexanes]; clear oil; $[\alpha]_{\mathrm{D}}{ }^{23}-32.9\left(\mathrm{c} 0.42, \mathrm{CH}_{2} \mathrm{Cl}_{2}\right) ;{ }^{\prime} \mathrm{H} \mathrm{NMR}\left(300 \mathrm{MHz}, \mathrm{CDCl}_{3}\right) \delta 0.88$ $(\mathrm{t}, 3 \mathrm{H}, J=6.9 \mathrm{~Hz}), 1.24-1.28(\mathrm{~m}, 4 \mathrm{H}), 1.33-1.52(\mathrm{~m}, 4 \mathrm{H}), 1.54-1.70(\mathrm{~m}, 2 \mathrm{H}), 2.48(\mathrm{t}, 2 \mathrm{H}, J=7.5 \mathrm{~Hz}), 3.89$ (dddd, $1 \mathrm{H}, J=5.1,7.8,10.5,13.2 \mathrm{~Hz}), 4.27(\mathrm{dd}, 1 \mathrm{H}, J=6.9,9.0 \mathrm{~Hz}), 4.69(\mathrm{t}, 1 \mathrm{H}, J=9.0 \mathrm{~Hz}), 5.46(\mathrm{dd}, 1 \mathrm{H}, J$ $=6.9,9.0 \mathrm{~Hz}), 7.03-7.05(\mathrm{~m}, 2 \mathrm{H}), 7.18-7.37(\mathrm{~m}, 8 \mathrm{H}) ;{ }^{13} \mathrm{C} \mathrm{NMR}\left(75 \mathrm{MHz}, \mathrm{CDCl}_{3}\right) \delta 13.9,22.8,28.5,29.5$, $31.5,32.4,35.8,42.5,57.8,69.6,125.6,126.0,128.2,128.3,128.7,129.1,139.2,142.1,153.4,176.4$; IR (thin film) $\mathrm{cm}^{-1} 2955(\mathrm{~m}), 2942(\mathrm{~m}), 2862(\mathrm{w}), 1782(\mathrm{~s}), 1703$ (s); mass spectrum (APCI): $\mathrm{m} / \mathrm{e}$ (\% relative intensity) $380(100)(\mathrm{M}+\mathrm{H})^{+}, 217(22), 189$ (73), $164(49), 87$ (41).

\section{Homo Allenyl Alcohol 45}

$\mathrm{R}_{\mathrm{f}}=0.39$ [25\% EtOAc in hexanes]; clear oil; $[\alpha]_{\mathrm{D}}{ }^{23}-65.6\left(\mathrm{c} 0.39, \mathrm{CH}_{2} \mathrm{Cl}_{2}\right) ;{ }^{1} \mathrm{H} \mathrm{NMR}\left(500 \mathrm{MHz}, \mathrm{CDCl}_{3}\right) \delta 0.88$ $(\mathrm{t}, 3 \mathrm{H}, J=7.0 \mathrm{~Hz}), 0.90(\mathrm{t}, 3 \mathrm{H}, J=7.0 \mathrm{~Hz}), 1.26-1.38(\mathrm{~m}, 8 \mathrm{H}), 1.39-1.47(\mathrm{~m}, 4 \mathrm{H}), 1.97-2.04(\mathrm{~m}, 2 \mathrm{H}), 2.18-$ $2.23(\mathrm{~m}, 1 \mathrm{H}), 3.48(\mathrm{dd}, 1 \mathrm{H}, J=7.5,10.5 \mathrm{~Hz}), 3.58-3.61(\mathrm{~m}, 1 \mathrm{H}), 4.95(\mathrm{dq}, 1 \mathrm{H}, J=3.0,6.5 \mathrm{~Hz}), 5.17(\mathrm{dq}, 1$ 
(C) 2003 American Chemical Society, Org. Lett., Frederick ol030061c Supporting Info Page 7

$\mathrm{H}, J=2.0,6.5, \mathrm{~Hz}) ;{ }^{13} \mathrm{C} \mathrm{NMR}\left(75 \mathrm{MHz}, \mathrm{CDCl}_{3}\right) \delta 13.9,22.4,22.6,28.7,28.8,29.2,30.8,31.2,42.4,66.1,91.7$, 92.0, 204.0 (missing 1 signal due to overlap of the terminal allene carbons); IR (thin film) $\mathrm{cm}^{-1} 3332$ (brs), 2963 $(\mathrm{m}), 2932(\mathrm{~s}), 2857(\mathrm{~m})$; mass spectrum $(\mathrm{APCI}): \mathrm{m} / \mathrm{e}$ (\% relative intensity) $211(100)(\mathrm{M}+\mathrm{H})^{+}, 193(27), 113$ (41), 85 (17).

Homo Allenyl Alcohol 46

$\mathrm{R}_{\mathrm{f}}=0.32\left[25 \%\right.$ EtOAc in hexanes]; clear oil; $[\alpha]_{\mathrm{D}}{ }^{23}+40.4\left(\mathrm{c} 0.46, \mathrm{CH}_{2} \mathrm{Cl}_{2}\right) ;{ }^{1} \mathrm{H} \mathrm{NMR}\left(500 \mathrm{MHz}, \mathrm{CDCl}_{3}\right) \delta 0.91$ $(\mathrm{t}, 3 \mathrm{H}, J=7.0 \mathrm{~Hz}), 1.32-1.56(\mathrm{~m}, 6 \mathrm{H}), 2.35-2.42(\mathrm{~m}, 1 \mathrm{H}), 3.60(\mathrm{t}, 1 \mathrm{H}, J=7.0 \mathrm{~Hz}), 3.65-3.70(\mathrm{~m}, 1 \mathrm{H}), 5.49$ $(\mathrm{t}, 1 \mathrm{H}, J=6.5 \mathrm{~Hz}), 6.22(\mathrm{dd}, 1 \mathrm{H}, J=2.0,6.5 \mathrm{~Hz}), 7.19-7.39(\mathrm{~m}, 5 \mathrm{H}) ;{ }^{13} \mathrm{C} \mathrm{NMR}\left(75 \mathrm{MHz}, \mathrm{CDCl}_{3}\right) \delta 13.9$, 22.6, 29.3, 30.8, 42.4, 66.1, 95.2, 96.3, 126.4, 126.7, 128.5, 135.6, 204.3; IR (thin film) $\mathrm{cm}^{-1} 3340$ (brs), 2961 $(\mathrm{m}), 2935(\mathrm{~s}), 2861(\mathrm{~m})$; mass spectrum (APCI): m/e (\% relative intensity) $217(93)(\mathrm{M}+\mathrm{H})^{+}, 199(12), 143$ (17), $117(15), 91(100)$.

Homo Allenyl Alcohol 47

$\mathrm{R}_{\mathrm{f}}=0.50\left[25 \%\right.$ EtOAc in hexanes]; clear oil; $[\alpha]_{\mathrm{D}}{ }^{23}+20.0\left(\mathrm{c} 0.10, \mathrm{CH}_{2} \mathrm{Cl}_{2}\right) ;{ }^{1} \mathrm{H}$ NMR $\left(500 \mathrm{MHz}, \mathrm{CDCl}_{3}\right) \delta 0.86-$ $0.92(\mathrm{~m}, 6 \mathrm{H}), 1.30-1.37(\mathrm{~m}, 8 \mathrm{H}), 1.47-1.56(\mathrm{~m}, 3 \mathrm{H}), 1.72-1.86(\mathrm{~m}, 1 \mathrm{H}), 2.12(\mathrm{q}, 2 \mathrm{H}, J=7.0 \mathrm{~Hz}), 2.78$ (quintet, $1 \mathrm{H}, J=6.0 \mathrm{~Hz}), 3.64-3.70(\mathrm{~m}, 2 \mathrm{H}), 5.60(\mathrm{dt}, 1 \mathrm{H}, J=1.5,7.0 \mathrm{~Hz}), 7.19-7.22(\mathrm{~m}, 1 \mathrm{H}), 7.30-7.34(\mathrm{~m}$, $2 \mathrm{H}), 7.40-7.42(\mathrm{~m}, 2 \mathrm{H}) ;{ }^{13} \mathrm{C}$ NMR $\left(75 \mathrm{MHz} \mathrm{CDCl}_{3}\right) \delta 13.9,22.5,22.8,24.8,29.0,29.3,30.6,31.3,37.7,62.9$, 65.6, 95.8, 126.2, 128.1, 128.3, 131.5, 203.1 (missing 1 signal); IR (thin film) $\mathrm{cm}^{-1} 3320$ (brs), 2960 (m), 2940 $(\mathrm{s}), 2870(\mathrm{~m})$; mass spectrum (APCI): m/e (\% relative intensity) $287(100)(\mathrm{M}+\mathrm{H})^{+}, 269(68), 257(39), 187$ (96), $117(86), 87(38)$.

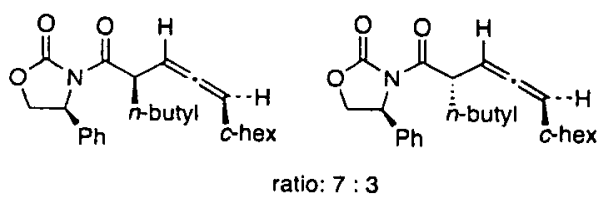

$\mathrm{R}_{\mathrm{f}}=0.43$ [25\% EtOAc in Hexanes]; clear oil; ' ${ }^{\mathrm{H}} \mathrm{NMR}\left(500 \mathrm{MHz}, \mathrm{CDCl}_{3}\right)$ Major isomer: $\delta 0.86-1.02(\mathrm{~m}, 5 \mathrm{H})$, $1.06-1.20(\mathrm{~m}, 2 \mathrm{H}), 1.24-1.36(\mathrm{~m}, 4 \mathrm{H}), 1.49-1.63(\mathrm{~m}, 6 \mathrm{H}), 1.70-1.88(\mathrm{~m}, 3 \mathrm{H}), 4.28(\mathrm{dd}, 1 \mathrm{H}, J=4.0,9.0 \mathrm{~Hz})$, $4.34(\mathrm{dq}, 1 \mathrm{H}, J=2.0,6.5 \mathrm{~Hz}), 4.67(\mathrm{t}, 1 \mathrm{H}, J=9.0 \mathrm{~Hz}), 5.20(\mathrm{dt}, 1 \mathrm{H}, J=2.0,6.0 \mathrm{~Hz}), 5.26-5.31(\mathrm{~m}, 1 \mathrm{H}), 5.43$ $(\mathrm{dd}, 1 \mathrm{H}, J=4.0,9.0 \mathrm{~Hz}), 7.26-7.40(\mathrm{~m}, 5 \mathrm{H})$; Minor Isomer: $\delta 0.86-1.02(\mathrm{~m}, 5 \mathrm{H}), 1.06-1.20(\mathrm{~m}, 2 \mathrm{H}), 1.24-$ $1.36(\mathrm{~m}, 4 \mathrm{H}), 1.49-1.63(\mathrm{~m}, 6 \mathrm{H}), 1.70-1.88(\mathrm{~m}, 3 \mathrm{H}), 4.26(\mathrm{dd}, 1 \mathrm{H}, J=4.0,9.0 \mathrm{~Hz}), 4.34(\mathrm{dq}, 1 \mathrm{H}, J=2.0,6.5$ $\mathrm{Hz}), 4.67(\mathrm{t}, 1 \mathrm{H}, J=9.0 \mathrm{~Hz}), 4.96(\mathrm{dt}, 1 \mathrm{H}, J=2.0,6.5 \mathrm{~Hz}), 5.23-5.26(\mathrm{~m}, 1 \mathrm{H}), 5.45(\mathrm{dd}, 1 \mathrm{H}, J=4.0,9.0 \mathrm{~Hz})$, 7.26-7.40 (m, $5 \mathrm{H}$ ); IR (thin film) $\mathrm{cm}^{-1} 2924(\mathrm{~m}), 2859(\mathrm{w}), 1785(\mathrm{~s}), 1707(\mathrm{~s}), 1457(\mathrm{~m}), 1379(\mathrm{~m})$; mass spectrum (APCI): $\mathrm{m} / \mathrm{e}$ (\% relative intensity) $382(66)(\mathrm{M}+\mathrm{H})^{+}, 219(100), 191(6), 164(8), 120(9)$. 